LBL-38585

March 26, 1996

\title{
Anomalous Transverse Distribution of Pions as a signal for the production of DCC's
}

\author{
F. Cooper, ${ }^{1}$, Y. Kluger, ${ }^{2}$ \\ E. Mottola, ${ }^{1}$ \\ ${ }^{1}$ Theoretical Division \\ Los Alamos National Laboratory \\ Los Alamos, New Mexico 87545, USA \\ ${ }^{2}$ Nuclear Science Division \\ Lawrence Berkeley Laboratory \\ Berkeley, CA 94720, USA
}

\begin{abstract}
We give evidence that the production of DCC's during a nonequilibrium phase transition can lead to an anomalous transverse distribution of secondary pions when compared to a more conventional boost invariant hydrodynamic flow in local thermal equilibrium. Our results pertain to the linear $\sigma$ model,treated in leading order in large$N$, in a boost invariant approximation. We also show that the interpolating number density of the field theory calculation plays the role of a classical relativistic phase space number distribution in determining the momentum distribution of pions in the center of mass frame.
\end{abstract}




\section{Introduction}

Recently there has been a growing interest in the possibility of producing disoriented chiral condensates (DCC's) in a high energy collision [1, 2, 3]. This idea was first proposed to explain CENTAURO events in cosmic ray emperiments where there was a deficit of neutral pions [4]. It was proposed that a nonequilibrium chiral phase transition such as a quench might lead to regions of DCC [3].

In a previous paper [5], we discussed in detail the time evolution of a non-equilibrium chiral phase transition in the linear $\sigma$ model in the leading order in large- $\mathrm{N}$ approximation. We showed in that paper that the expansion into the vacuum of the initial energy distribution led to rapid cooling. This caused the system, initially in quasi local thermal equilibrium to progress from the unbroken chiral symmetry phase to the broken symmetry phase vacuum. This expansion is accompanied by the exponential growth of low momentum modes for short periods of proper time for a range of initial conditions. This exponential growth of long wave length modes is the mechanism for the production of disordered chiral condensates. Thus the production of DCC's results in an enhancement of particle production in the low momentum domain. Whether such an instability occurs depends on the size of the initial fluctuation from the initial thermal distribution. The relevant momenta for which this exponential growth occurs are the transverse momenta and the momenta $k_{\eta}=-E z+t p$ conjugate to the fluid rapidity variable $\eta=\tanh ^{-1}(z / t)$. We also found that the distribution of particles in these momenta had more length scales than found in local thermal equilibrium. When there is local thermal equilibrium, the length scales are the mass of the pion and the temperature which is related to the changing energy density, both of which depend on the proper time $\tau$.

In this paper we reexpress our previous results in terms of the physically measurable transverse distribution of particles in the collision center of mass frame. We show that whenever DCC's are produced there is a noticable distortion of the transverse spectrum, namely an enhancement of particles at low transverse momentum, when compared to a local equilibrium evolution. We will consider two cases, one in which there is exponential growth of low momentum modes due to the effective pion mass going negative during the expansion, and one where the initial fluctuations do not lead to this exponential growth. Both situations will be compared to a purely hydrodynamical 
boost invariant calculation based on local thermal equilibrium. The idea that the production of DCC's might lead to a distortion of the transverse distribution of pions is also found in the work of Gavin and collaborators [6]. However, our calculation is more realistic in that we exactly solve the field theory in a large $N$ approximation in the boost invariant approximation, using the expansion of the plasma as the cooling mechanism for producing instabilities. In determining the actual spectra of secondaries, we find that the adiabatic number operator of our large- $\mathrm{N}$ calculation replaces the relativistic phase space density $g(x, p)$ of classical transport theory in determining the distribution of pariticles in rapidity and transverse momentum. This makes it easy to compare our results with a standard hydrodynamical calculation in the boost invariant approximation which assumes the final pions are in local thermal equilibrium in the comoving frame.

The model we use to discuss the chiral phase transition is the linear sigma model described by the Lagrangian:

$$
L=\frac{1}{2} \partial \Phi \cdot \partial \Phi-\frac{1}{4} \lambda\left(\Phi \cdot \Phi-v^{2}\right)^{2}+H \sigma .
$$

The mesons form an $O(4)$ vector $\Phi=\left(\sigma, \pi_{i}\right)$ This can be written in an alternative form by introducing the composite field: $\chi=\lambda\left(\Phi \cdot \Phi-v^{2}\right)$.

$$
L_{2}=-\frac{1}{2} \phi_{i}(\square+\chi) \phi_{i}+\frac{\chi^{2}}{4 \lambda}+\frac{1}{2} \chi v^{2}+H \sigma
$$

The effective action to leading order in large $N$ is given by [5]

$$
\begin{gathered}
\Gamma[\Phi, \chi]=\int d^{4} x\left[L_{2}(\Phi, \chi, H)+\frac{i}{2} N \operatorname{tr} \ln G_{0}^{-1}\right] \\
G_{0}^{-1}(x, y)=i[\square+\chi(x)] \delta^{4}(x-y)
\end{gathered}
$$

Varying the action we obtain:

$$
[\square+\chi(x)] \pi_{i}=0 \quad[\square+\chi(x)] \sigma=H,
$$

where here and in what follows, $\pi_{i}, \sigma$ and $\chi$ refer to expectation values. Varying the action we obtain

$$
\chi=-\lambda v^{2}+\lambda\left(\sigma^{2}+\pi \cdot \pi\right)+\lambda N G_{0}(x, x)
$$


If we assume boost invariant kinematics [0] which result in flat rapidity distributions, then the expectation value of the energy density is only a function of the proper time. The natural coordinates for boost invariant $(v=z / t)$ hydrodynamical flow are the fluid proper time $\tau$ and the fluid rapidity $\eta$ defined as

$$
\tau \equiv\left(t^{2}-z^{2}\right)^{1 / 2}, \quad \eta \equiv \frac{1}{2} \log \left(\frac{t-z}{t+z}\right) .
$$

To implement boost invariance we assume that mean (expectation) values of the fields $\Phi$ and $\chi$ are functions of $\tau$ only. We then get the equations:

$$
\begin{aligned}
& \tau^{-1} \partial_{\tau} \tau \partial_{\tau} \Phi_{i}(\tau)+\chi(\tau) \Phi_{i}(\tau)=H \delta_{i 1} \\
& \chi(\tau)=\lambda\left(-v^{2}+\Phi_{i}^{2}(\tau)+N G_{0}(x, x ; \tau, \tau)\right),
\end{aligned}
$$

To determine the Green's function $G_{0}\left(x, y ; \tau, \tau^{\prime}\right)$ we introduce the auxiliary quantum field $\phi(x, \tau)$ which obeys the sourceless equation:

$$
\begin{gathered}
\left(\tau^{-1} \partial_{\tau} \tau \partial_{\tau}-\tau^{-2} \partial_{\eta}^{2}-\partial_{\perp}^{2}+\chi(x)\right) \phi(x, \tau)=0 . \\
G_{0}\left(x, y ; \tau, \tau^{\prime}\right) \equiv<T\left\{\phi(x, \tau) \phi\left(y, \tau^{\prime}\right)\right\}>
\end{gathered}
$$

We expand the quantum fields in an orthonormal basis:

$$
\phi\left(\eta, x_{\perp}, \tau\right) \equiv \frac{1}{\tau^{1 / 2}} \int\left[d^{3} \mathrm{k}\right]\left(\exp (i k x) f_{\mathrm{k}}(\tau) a_{\mathrm{k}}+\text { h.c. }\right)
$$

where $k x \equiv k_{\eta} \eta+\vec{k}_{\perp} \vec{x}_{\perp},\left[d^{3} \mathrm{k}\right] \equiv d k_{\eta} d^{2} k_{\perp} /(2 \pi)^{3}$. The mode functions and $\chi$ obey:

$$
\begin{gathered}
\ddot{f}_{\mathrm{k}}+\left(\frac{k_{\eta}^{2}}{\tau^{2}}+\vec{k}_{\perp}^{2}+\chi(\tau)+\frac{1}{4 \tau^{2}}\right) f_{\mathrm{k}}=0 . \\
\chi(\tau)=\lambda\left(-v^{2}+\Phi_{i}^{2}(\tau)+\frac{1}{\tau} N \int\left[d^{3} \mathrm{k}\right]\left|f_{\mathrm{k}}(\tau)\right|^{2}\left(1+2 n_{\mathrm{k}}\right)\right) .
\end{gathered}
$$

We notice that when $\chi$ goes negative, the low momentum modes with

$$
\frac{k_{\eta}^{2}+1 / 4}{\tau^{2}}+\vec{k}_{\perp}^{2}<|\chi|
$$

grow exponentially. However these modes then feed back into the $\chi$ equation and this exponential growth then gets damped. It is these growing modes 
that lead to the possiblity of growing domains of DCC's as well as a modification of the low momentum distribution of particles from a thermal one. The parameters of the model are fixed by physical data. The PCAC condition is

$$
\partial_{\mu} A_{\mu}^{i}(x) \equiv f_{\pi} m_{\pi}^{2} \pi^{i}(x)=H \pi^{i}(x) .
$$

In the vacuum state $\chi_{0} \sigma_{0}=m_{\pi}^{2} \sigma_{0}=H$, so that $\sigma_{0}=f_{\pi}=92.5 \mathrm{MeV}$. The vacuum gap equation is

$$
m_{\pi}^{2}=-\lambda v^{2}+\lambda f_{\pi}^{2}+\lambda N \int_{0}^{\Lambda}\left[d^{3} \mathrm{k}\right] \frac{1}{2 \sqrt{k^{2}+m_{\pi}^{2}}}
$$

This leads to the mass renormalized gap equation:

$$
\begin{aligned}
& \chi(\tau)-m_{\pi}^{2}=-\lambda f_{\pi}^{2}+\lambda \Phi_{i}^{2}(\tau) \\
& +\frac{\lambda}{\tau} N \int\left[d^{3} \mathrm{k}\right]\left\{\left|f_{\mathrm{k}}(\tau)\right|^{2}\left(1+2 n_{\mathrm{k}}\right)-\frac{1}{2 \sqrt{k^{2}+m^{2}}}\right\} .
\end{aligned}
$$

$\lambda$ is chosen to fit low energy scattering data as discussed in [5].

If we assume that the inititially (at $\left.\tau_{0}=1\right)$ the system is in local thermal equilibrium in a comoving frame we have

$$
n_{k}=\frac{1}{e^{\beta_{0} E_{k}^{0}}-1}
$$

where $\beta_{0}=1 / T_{0}$ and $E_{k}^{0}=\sqrt{\frac{k_{\eta}^{2}}{\tau_{0}^{2}}+\vec{k}_{\perp}^{2}+\chi\left(\tau_{0}\right)}$.

The initial value of $\chi$ is determined by the equilibrium gap equation for an initial temperature of $200 \mathrm{MeV}$ and is $.7 \mathrm{fm}^{-2}$ and the initial value of $\sigma$ is just $\frac{H}{\chi_{0}}$. The phase transition in this model occurs at a critical temperature of $160 \mathrm{MeV}$. To get into the unstable domain, we then introduce fluctuations in the time derivative of the classical field. We varied the value of the initial proper time derivative of the sigma field expectation value and found that for $\tau_{0}=1 \mathrm{fm}$ there is a narrow range of initial values that lead to the growth

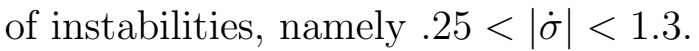

Figure one displays the results of the numerical simulation for the evolution of the system (1.8)-(1.9). We display the auxiliary field $\chi$ in units of $\mathrm{fm}^{-2}$ and the proper time in units of $\mathrm{fm}\left(1 \mathrm{fm}^{-1}=197 \mathrm{MeV}\right)$ for two simulations, one with an instability $\left(\left.\dot{\sigma}\right|_{\tau_{0}}=-1\right)$ and one without $\left(\left.\dot{\sigma}\right|_{\tau_{0}}=0\right)$. 
We notice that for both initial conditions, the system eventually settles down to the broken symmetry vacuum result as a result of the expansion.

To determine the spectrum of particles we introduce the interpolating number density which is defined by expanding the fields in terms of mode functions $f_{k}^{0}$ which are first order in an adiabatic expansion of the mode equation.

$$
f_{k}^{0}=\frac{e^{-i y_{k}(\tau)}}{\sqrt{2 \omega_{k}}} ; \quad d y_{k} / d t=\omega_{k},
$$

where $\omega_{\mathrm{k}}(\tau) \equiv\left(k_{\eta}^{2} / \tau^{2}+\vec{k}_{\perp}^{2}+\chi(\tau)\right)^{1 / 2}$. This leads to the alternative expansion of the fields:

$$
\phi\left(\eta, x_{\perp}, \tau\right) \equiv \frac{1}{\tau^{1 / 2}} \int\left[d^{3} \mathrm{k}\right]\left(\exp (i k x) f_{\mathrm{k}}^{0}(\tau) a_{\mathrm{k}}(\tau)+h . c .\right)
$$

The two sets of creation and annihilation operators are connected by a Bogoliubov transformation:

$$
a_{k}(\tau)=\alpha(k, \tau) a_{k}+\beta(k, \tau) a_{-k}^{\dagger} .
$$

$\alpha$ and $\beta$ can be determined from the exact time evolving mode functions via:

$$
\begin{aligned}
\alpha(k, \tau) & =i\left(f_{k}^{0 *} \frac{\partial f_{k}}{\partial \tau}-\frac{\partial f_{k}^{0 *}}{\partial \tau} f_{k}\right) \\
\beta(k, \tau) & =i\left(f_{k}^{0} \frac{\partial f_{k}}{\partial \tau}-\frac{\partial f_{k}^{0}}{\partial \tau} f_{k}\right) .
\end{aligned}
$$

In terms of the initial distribution of particles $n_{0}(k)$ and $\beta$ we have:

$$
\begin{aligned}
n_{k}(\tau) & \equiv f\left(k_{\eta}, k_{\perp}, \tau\right)=<a_{k}^{\dagger}(\tau) a_{k}(\tau)> \\
& =n_{0}(k)+|\beta(k, \tau)|^{2}\left(1+2 n_{0}(k)\right) .
\end{aligned}
$$

$n_{k}(\tau)$ is the adiabatic invariant interpolating phase space number density which becomes the actual particle number density when interactions have ceased. When this happens the distribution of particles is

$$
f\left(k_{\eta}, k_{\perp}, \tau\right)=\frac{d^{6} N}{\pi^{2} d x_{\perp}^{2} d k_{\perp}^{2} d \eta d k_{\eta}} .
$$

We now need to relate this quantity to the physical spectra of particles measured in the lab. At late $\tau$ our system relaxes to the vacuum and $\chi$ becomes 
the square of the physical pion mass $\mathrm{m}^{2}$. We introduce the outgoing pion particle rapidity $y$ and $m_{\perp}=\sqrt{k_{\perp}^{2}+m^{2}}$ defined by the particle 4-momentum in the center of mass coordinate system

$$
k_{\mu}=\left(m_{\perp} \cosh y, k_{\perp}, m_{\perp} \sinh y\right)
$$

The boost that takes one from the center of mass coordinates to the comoving frame where the energy momentum tensor is diagonal is given by $\tanh \eta=$ $v=z / t$, so that one can define the "fluid" 4-velocity in the center of mass frame as

$$
u^{\mu}=(\cosh \eta, 0,0, \sinh \eta)
$$

We then find that the variable

$$
\omega_{k}=\sqrt{m_{\perp}^{2}+\frac{k_{\eta}^{2}}{\tau^{2}}} \equiv k^{\mu} u_{\mu}
$$

has the meaning of the energy of the particle in the comoving frame. The momenta $k_{\eta}$ that enters into the adiabatic phase space number density is one of two canoncial momenta to the variables defined by the coordinate transformation to fluid light cone variables. Namely the variables

$$
\tau=\left(t^{2}-z^{2}\right)^{1 / 2} \quad \eta=\frac{1}{2} \ln \left(\frac{t+z}{t-z}\right)
$$

have as their canonical momenta

$$
k_{\tau}=E t / \tau-k_{z} z / \tau \quad k_{\eta}=-E z+t k_{z}
$$

To show this we consider the metric $d s^{2}=d \tau^{2}-\tau^{2} d \eta^{2}$ and the free Lagrangian

$$
L=\frac{m}{2} g_{\mu \nu} \frac{d x^{\mu}}{d s} \frac{d x^{\nu}}{d s}
$$

Then we obtain for example

$$
\begin{aligned}
k_{\tau} & =m \frac{d \tau}{d s}=m\left[\left(\frac{\partial \tau}{\partial t}\right)_{z} \frac{d t}{d s}+\left(\frac{\partial \tau}{\partial z}\right)_{t} \frac{d z}{d s}\right] \\
& =\frac{E t-k_{z} z}{\tau}=k^{\mu} u_{\mu}
\end{aligned}
$$


The interpolating phase-space density $f$ of particles depends on $k_{\eta}, \mathbf{k}_{\perp}, \tau$, and it is found to be $\eta$-independent and given by eq. (1.17).

In order to obtain the physical particle rapidity and transverse momentum distribution, we change variables from $\left(\eta, k_{\eta}\right)$ to $(z, y)$ at a fixed $\tau$ where $y$ is the particle rapidity defined by (1.18). We have

$$
E \frac{d^{3} N}{d^{3} k}=\frac{d^{3} N}{\pi d y d k_{\perp}^{2}}=\int \pi d z d x_{\perp}^{2} J f\left(k_{\eta}, k_{\perp}, \tau\right)
$$

where the Jacobian $J$ is evaluated at a fixed proper time $\tau$

$$
\begin{aligned}
J & =\left|\begin{array}{cc}
\partial k_{\eta} / \partial y & \partial k_{\eta} / \partial z \\
\partial \eta / \partial y & \partial \eta / \partial z
\end{array}\right|=\left|\frac{\partial k_{\eta}}{\partial y} \frac{\partial \eta}{\partial z}\right| \\
& =\frac{m_{\perp} \cosh (\eta-y)}{\cosh \eta}=\left.\frac{\partial k_{\eta}}{\partial z}\right|_{\tau} .
\end{aligned}
$$

We also have

$$
k_{\tau}=m_{\perp} \cosh (\eta-y) ; \quad k_{\eta}=-\tau m_{\perp} \sinh (\eta-y) .
$$

Calling the integration over the transverse dimension the effective transverse size of the colliding ions $A_{\perp}$ we then obtain that:

$$
\frac{d^{3} N}{\pi d y d k_{\perp}^{2}}=A_{\perp} \int d k_{\eta} f\left(k_{\eta}, k_{\perp}, \tau\right)
$$

This quantity is independent of $y$ which is a consequence of the assumed boost invariance.

In a hydrodynamical model of heavy ion collisions [7], the final spectra of pions is given by a combination of the fluid flow and a local thermal equilibrium distribution in the comoving frame. One calculates this spectra at the critical temperature $T_{c}(x, t)$ when the energy density goes below $\epsilon_{c}=\frac{1}{(\hbar / m c)^{3}}$. This defines the breakup surface, after which the particles no longer interact so that this distibution is frozen at that temperature. For an ultrarelativistic gas of pions, this occurs when $T_{c} \approx m$. The covariant form for the spectra of particles is given by the Cooper- Frye formula [8]:

$$
E \frac{d^{3} N}{d^{3} k}=\frac{d^{3} N}{\pi d k_{\perp}^{2} d y}=\int g(x, k) k^{\mu} d \sigma_{\mu}
$$


Here $g(x, k)$ is the single particle relativistic phase space distribution function. When there is local thermal equilibrium of pions at a comoving temperature $T_{c}(\tau)$ one has

$$
g(x, k)=\frac{g_{\pi}}{(2 \pi)^{3}}\left\{\exp \left[k^{\mu} u_{\mu} / T_{c}\right]-1\right\}^{-1} .
$$

In the boost invariant approximation, the temperature is only a function of the proper time $\tau$ and the break up surface is a constant $\tau$ surface:

$$
d \sigma^{\mu}=A_{\perp}(d z, 0,0, d t)=A_{\perp} d \eta(\cosh \eta, 0,0, \sinh \eta)
$$

where $A_{\perp}$ is the effective transverse size of the system following the heavy ion collision. Therefore we obtain the relationship that

$$
k^{\mu} d \sigma_{\mu}=A_{\perp} m_{\perp} \tau \cosh (\eta-y)=A_{\perp}\left|d k_{\eta}\right|
$$

Thus we can rewrite our expression for the field theory particle spectra as

$$
\frac{d^{3} N}{\pi d y d k_{\perp}^{2}}=A_{\perp} \int d k_{\eta} f\left(k_{\eta}, k_{\perp}, \tau\right)=\int f\left(k_{\eta}, k_{\perp}, \tau\right) k^{\mu} d \sigma_{\mu}
$$

where in the second integration we keep $y$ and $\tau$ fixed. This shows that the Cooper-Frye formula for the final spectra of secondaries, which was derived from classical transport theory, also applies in field theory when one has a gaussian density matrix as in the large- $\mathrm{N}$ approximation. It also reconfirms the idea that the interpolating phase space number density plays the role of a classical transport phase space density function, as was found in our calculation of pair production from strong electric fields 9]

In Figures 2 and 3 we compare the boost invariant hydrodynamical result for the transverse momentum distribution using critical temperatures of $T_{c}=140,200 \mathrm{MeV}$ to the two nonequilibrium cases represented in figure 1. Figure 2 pertains to the initial condition $\left.\dot{\sigma}\right|_{\tau_{0}}=-1$ In this case there is a regime where the effective mass becomes negative and we see a noticable enhancement of the low transverse momentum spectra. We have normalized both results to give the same total center of mass energy $E_{c m}$. Figure 3 corresponds to the initial condition $\left.\dot{\sigma}\right|_{\tau_{0}}=0$. Here we notice that there is a little enhancement at low transverse momenta. 
In conclusion, we see that a non-equilibrium phase transition taking place during a time evolving quark-gluon or hadronic plasma can lead to an enhancement of the low momentum transverse momentum distribution. In particular, if a Centauro type event is not accompanied by such an enhancement one would be suspicious of ascribing this event to the production of disoriented chiral condensates as a result of a rapid quench.

\section{References}

[1] A. Anselm, Phys. Lett. B217, 169 (1989); A. Anselm and M. Ryskin, Phys. Lett. B226, 482 (1991).

[2] J.D. Bjorken, Int. Jour. of Mod. Phys.A7, 4189 (1992); Acta Phys. Pol. B23 561 (1992).

[3] K. Rajagopal and F. Wilczek, Nucl. Phys. B379, 395 (1993); S. Gavin, A. Gocksch and R.D. Pisarski, Phys. Rev. Lett. 72,2143 (1994)

[4] C.M.G. Lattes, Y. Fujimoto and S. Hasegawa, Phys. Rep. 65, 151 (1980).

[5] F. Cooper, Y.Kluger, E.Mottola, J.P. Paz, Phys. Rev. D51,2377 (1995).

[6] Sean Gavin, hep-ph/9407368 and references therein.

[7] F. Cooper, G. Frye and E. Schonberg, Phys. Rev. D11 192 (1975); J.D. Bjorken, Phys. Rev. D27, 140 (1983)

[8] F. Cooper and G. Frye Phys. Rev. D10 ,186 (1974)

[9] F. Cooper, J. M. Eisenberg, Y. Kluger, E. Mottola, and B. Svetitsky, Phys Rev. D 48190 (1993)

FIGURE 1. Proper time evolution of the $\chi$ field for two different initial conditions $(\dot{\sigma}(1)=0$ and $\dot{\sigma}(1)=-1)$ with $f_{\pi}=92.5 \mathrm{MeV}$.

FIGURE 2. Single particle distribution $k_{\perp} \frac{E d N}{d^{3} k}$ vs. $k_{\perp}$ in the mean field approximation for the initial condition $\dot{\sigma}(1)=-1$ compared to two different hydrodynamical calculations with two different breakup temperatures: $T_{c}=$ $m_{\pi}$ and $T_{c}=1.4 m_{\pi}$. We have normalized all the curves to give the same center of mass energy. 
FIGURE 3. Same comparison as in fig. 2 but with the mean field approximation for the initial condition $\dot{\sigma}(1)=0$. 


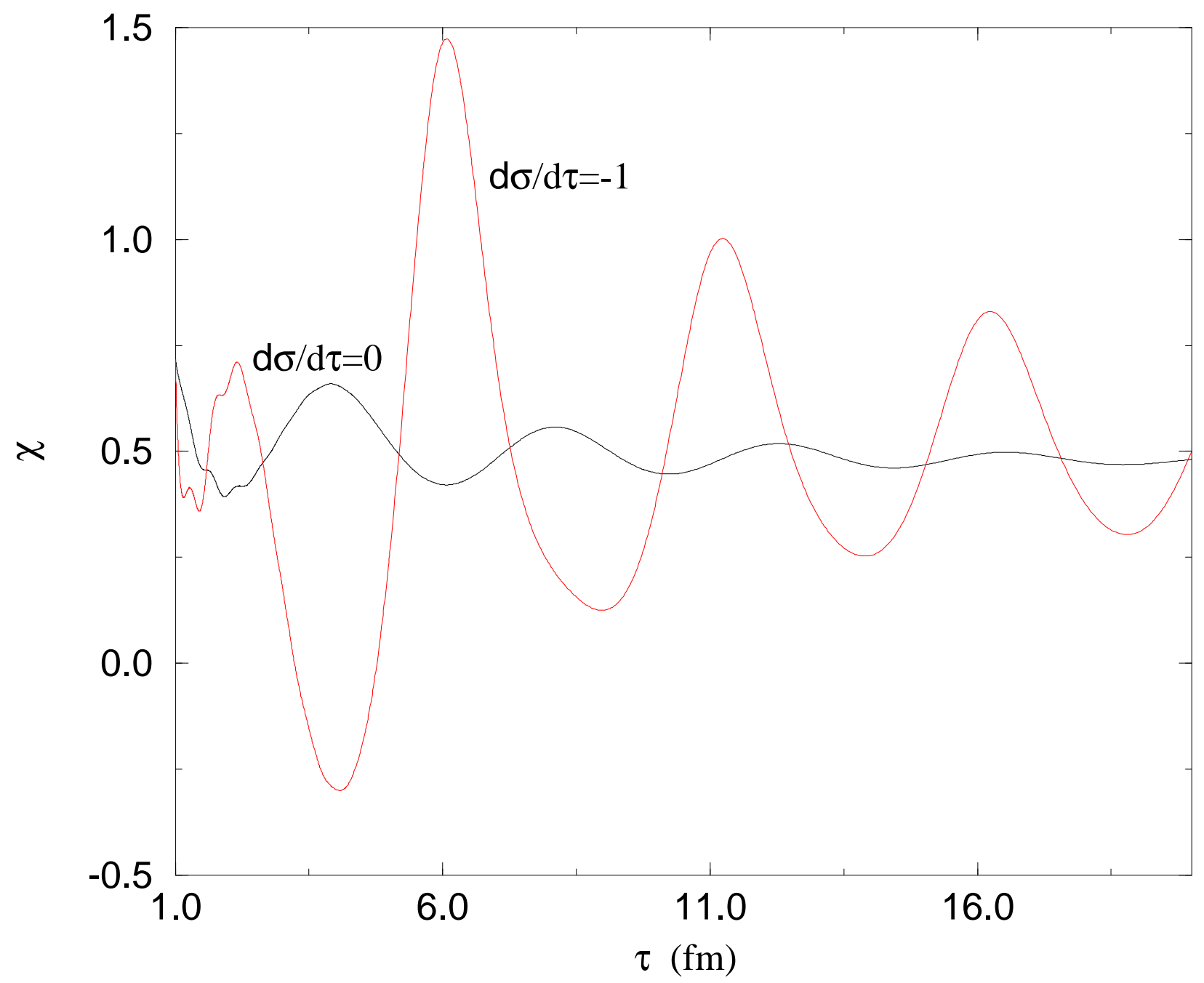




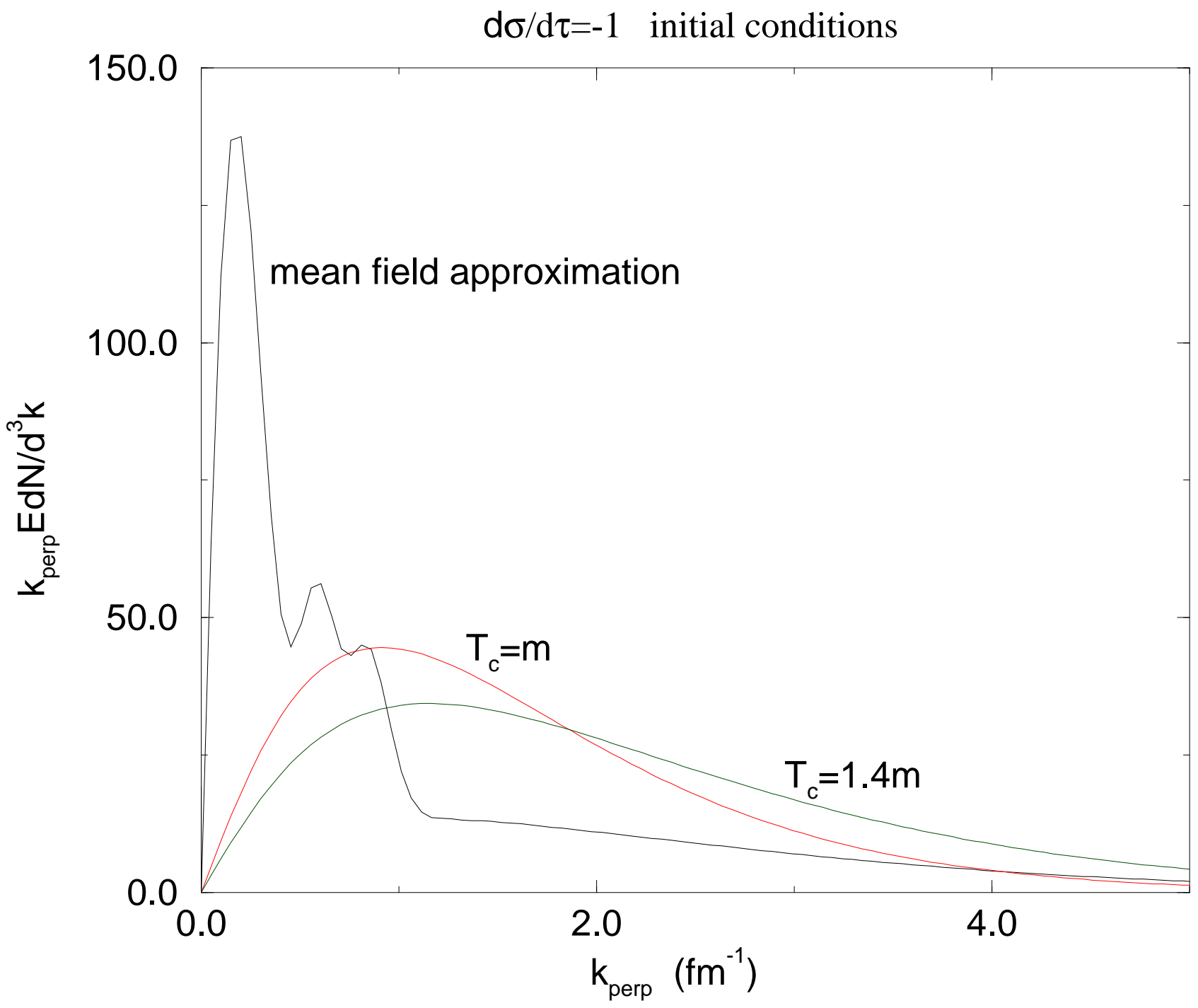




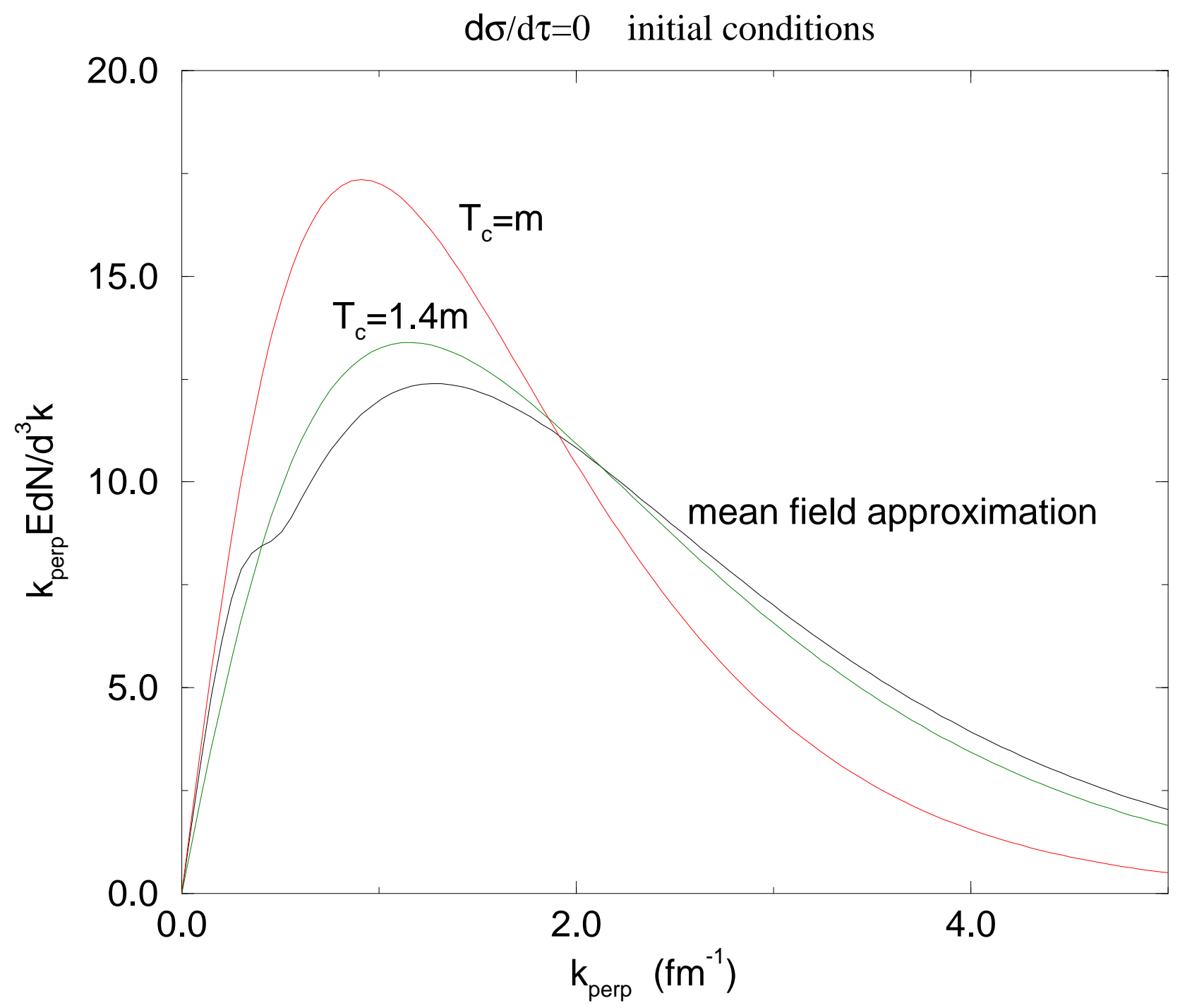

